\title{
Patient mix optimisation and stochastic resource requirements: A case study in cardiothoracic surgery planning
}

\author{
Ivo Adan • Jos Bekkers • Nico Dellaert • \\ Jan Vissers $\cdot$ Xiaoting Yu
}

Received: 15 December 2007 / Accepted: 21 August 2008/Published online: 4 October 2008

(C) The Author(s) 2008. This article is published with open access at Springerlink.com

\begin{abstract}
Cardiothoracic surgery planning involves different resources such as operating theatre time, beds, IC beds and nursing staff. In the daily practice of the Thorax Centre case study setting, the planning focuses on optimal use of operating theatre time, though the performance of the Thorax Centre as a whole is often more limited by other resources. For operating theatres a master surgical schedule is used to allocate operating theatre resources at tactical level for a longer period. Operational schedules at weekly level are derived from this master schedule. Within cardiothoracic surgery different categories of patients can be distinguished based on their requirement of resources. The mix of patients operated is, therefore, an important decision variable for the Thorax Centre to manage the use of these resources. In this paper we will consider the
\end{abstract}

Submitted for ORAHS2007 special issue in HCMS, version 14th July 2008

I. Adan $\cdot X$. Yu

Department of Mathematics and Computer Sciences,

Eindhoven University of Technology,

Eindhoven, The Netherlands

J. Bekkers

Department of Cardiothoracic Surgery,

Erasmus University Medical Centre Rotterdam,

Rotterdam, The Netherlands

N. Dellaert $\cdot$ J. Vissers

Department of Technology Management,

Eindhoven University of Technology,

Eindhoven, The Netherlands

J. Vissers $(\bowtie)$

Institute of Health Policy and Management,

Erasmus University Medical Centre Rotterdam,

Rotterdam, The Netherlands

e-mail: Vissers@bmg.eur.nl planning problem at the tactical level to generate a master surgical schedule that realises a given target of patient throughput and optimises an objective function for the utilisation of resources. The problem can be mathematically approached by mixed integer linear programming, which we already demonstrated in a previous paper. The specific topic of the current paper is to investigate the influence of using a stochastic instead of a deterministic length of stay. We will discuss the new mathematical model developed for this planning problem. The results obtained by the model indicate that we can generate master surgical schedules with a better performance on target utilization levels of resources by considering the stochastic length of stay.

Keywords Operating theatre planning .

Admission planning - Patient mix - Stochastic length of stay Resource allocation $\cdot$ Integer linear programming

\section{Introduction}

Hospital admission planning refers to the operational planning of patients who need to be admitted as inpatients to a hospital [1]. Patients can be classified as elective, urgent or emergency. Elective patients do not have to be treated immediately and can therefore be put on a waiting list, to be called when it is their turn, with just a vague notion of the actual admission moment. Alternatively, elective patients can be given an appointment for admission. Urgent patients need to be admitted at short notice, which is usually as soon as a bed becomes available. Emergency patients need to be admitted immediately.

There is an increasing interest for research on admission planning in hospitals and related topics of operating theatre planning, bed planning and waiting lists. This is due to the 
awareness of hospital management that improved logistics performance in planning results in shorter access, waiting and throughput time, and is an effective tool to compete with other hospitals. It is a response to the paradigm change in the service philosophy of hospitals, i.e. from optimising the use of scarce resources to finding a balance between the quality of service delivery and the efficiency in the use of resources. This change in service philosophy was necessary because patients nowadays do not accept long waiting times, and service quality aspects in health care delivery play an important role in patient satisfaction. More-over, elective patients can choose between hospitals for their surgery since the urgency of their procedures is low, so it is important for hospitals to be competitive.

To compare different approaches to hospital admission planning Vissers, Adan and Dellaert [2] use a framework that distinguishes different levels within admission planning: the admission service concept at strategic level (what is the philosophy behind and what are the objectives for admission planning), the admission policy at tactical level (what is the mix of patients to be admitted and what amount of resources is required), and admission scheduling rules at operational level (what is the best scheduling of individual patients).

They furthermore distinguish a number of features of the approach followed, such as the scope of the resources included in the study (e.g. operating theatre resources, ward beds and IC beds, nursing staff and specialist capacity), the assumptions on stochastic behaviour of variables in the system (e.g. percentage of urgent admissions, length of stay, duration of surgery), and features of the setting investigated, such as the level of detail investigated (e.g. individual patients, groups of patients, specialty or hospital). These components of the framework for comparison of admission planning systems can be used to discuss the relevant literature, and to position our work.

Admission planning, waiting lists and operating theatre management have received much research attention, as well as in clinical management literature (e.g. Anesthesia \& Analgesia, Anesthesiology) as in management science literature (e.g. European Journal of Operational Research, Health Care Management Science). Gemmel and Van Dierdonck [3] provide an overview of research on admission planning. Many of the studies that are reported in [3] focus on improving the scheduling of admissions and resources at operational level. Smith-Daniels et al. [4] give already a warning in their extensive literature review on capacity management in hospitals that most admission scheduling systems only consider bed capacity and that most surgery planning systems only consider operating theatre capacity. This may lead to sub-optimal use of other resources such as nursing staff and intensive care capacity.

More recent studies on operating theatre planning take a multi-resource perspective on scheduling surgery patients.
Beliën and Demeulemeester [5] for instance, see development of effective operating room schedules as a three stage process: allocation of OR time to surgical specialties at strategic level, development of a master surgery schedule at tactical level, scheduling individual patients at operational level. According to Beliën and Demeulemeester a better performance of the master surgical schedule can result in an overall improvement of resource use of operating theatres, wards and intensive care, by taking into account the dependencies between these resources. Poorly developed master surgical schedules can result in variability in demand of beds and nursing staff at wards and intensive care. Their elaboration of this problem concentrated on the relationship between the master surgical schedule and the bed occupancy. Two types of mathematical approaches were followed: a Mixed Integer Program heuristic (MIP) and a simulated annealing heuristic (SA). The SA approach gave better results but the MIP approach required less computational effort and allowed according to the authors easier tuning to specific requirements of a setting by defining constraints or manipulating weights in the objective function. Van Oostrum et al. [6] also look at cyclic master scheduling for operating rooms and the impact on succeeding departments. They use data on actual surgical procedures and their stochasticity and focus on the optimisation of the use of operating theatre resources.

Clinical management studies provide evidence that variability in scheduled surgical caseload can be reduced. McManus et al. [7] make a distinction between natural variability and artificial variability. Natural variability arises from uncertainty in patient arrivals, recovery time, etc. Artificial variability originates from poor scheduling policies. McManus et al. [7] investigated variability in an intensive care unit, and found that the scheduled patient flow was more variable than the patient flow resulting from emergencies. Especially systems operating near capacity may benefit from improving control over artificial variability by improving scheduling. McManus et al. [8] demonstrated in a follow up study that a queuing model approach may be used to accurately model the bed utilization in a larger intensive care unit operating near capacity.

Wachtel and Dexter [9] give warning that tactical increases in operating room (OR) block time for capacity planning should not be based on utilization. Tactical increases (at the level of annual decision making) should rather be based on contribution margins per OR hour, potential for growth, and limitations in scarce resources. They argue that this misunderstanding is caused by mixing up the tactical level where decisions are taken to expand or decrease capacity by allocating block time and the operational level where staffing levels are determined for anticipated workload. 
In this paper we focus on a planning issue at tactical level, i.e. the mix of patients that ideally need to be admitted at each day within a cyclical planning period (for instance 4 weeks) to optimise the use of resources, given an objective function and taking into account some restrictions in planning combinations of patients and availability of resources. The objective function is taken as the total absolute deviation of the utilization of the resources from their targets over all days of the planning cycle. The resources included are Medium Care (MC) beds, Intensive Care (IC) beds, operating theatre capacity and IC-nursing staff. They are considered as most critical for the problem due to limitations in availability. Mix of patients implies that there are different groups of patients, each with their own resource requirements. We are going to solve this problem at the aggregate level of patient groups and at the tactical level of the master schedule, as improvement of the master schedule should be the first step to take. We are aware that this is not the whole problem. The schedules at operational level - derived from the master schedule - will determine the actual performance of the system, expressed in waiting times for patients and cancellations of scheduled procedures, use of overtime for surgical sessions that overrun, and occupancy of resources. As we concentrate in this paper on the master schedule at tactical level with an anticipated volume of procedures that should balance demand, we can limit ourselves to expected performance on utilization of resources.

Previous work on the same subject $[10,11]$ involved modelling admission planning at tactical level with multiple resources and constraints, using data from general surgery and cardiothoracic surgery, but based on a deterministic length of stay on IC and wards corresponding to an estimate for the average. In this study we will investigate the impact of stochastic length of stay for IC and $\mathrm{MC}$ on the performance of the admission planning at tactical level and see what the impact is on the proposed admission profile for the planning period generated by the model. We will again use the setting of the Thorax Centre Rotterdam for comparison with the previous approach. Compared to the work by Beliën and Demeulemeester [5] and Van Oostrum et al. [6] we are less interested in finding the best mathematical solution. Instead, the aim in this paper is to offer mathematical support in a case setting where a cardiothoracic surgeon-planner wants to develop a planning approach for scheduling surgical patients that can provide information on the consequences of relevant resources and that can be implemented in practice.

The remainder of the paper is structured as follows. Section 2 provides information on the case study setting, elaborates on the planning problem, and ends with the research questions to be answered in this paper. Section 3 presents the new mathematical model that takes into account stochastic resource requirements. Section 4 illustrates the use of the model and the sensitivity with respect to the length of stay distribution. In Section 5 we will draw some conclusions and formulate recommendations for further work.

\section{Case study setting}

The Thorax Centre Rotterdam is an important department of the Erasmus University Medical Centre, providing care to patients suffering from malfunctions of the heart, lung and the intrathoracic vessels. The Department of Cardiothoracic Surgery performs surgery of coronary heart disease (coronary artery bypass grafting-CABG), surgery of valvular and congenital abnormalities in children and adults, lung operations as well as heart transplants. This department has a surgical staff of eight Cardio-Thoracic surgeons, trained to perform all adult cardiac and pulmonary operations, including heart transplants. Two surgeons are specialized in congenital heart operations. Specialists in Dutch university hospitals work on a salaried basis. There is not much competition between hospitals for this type of surgery as only a limited number of hospitals are allowed to perform cardiothoracic surgery, referral patterns are settled, sufficient capacity is available and waiting times of 68 weeks are considered as acceptable for elective procedures. This setting may differ from settings in other healthcare systems with more competition between hospitals and a fee for service salary system.

The patient flow of the Thorax Centre can be distinguished in scheduled patients (elective patients from the waiting list) and non-scheduled patients (emergency patients requiring immediate surgery). In this paper we only take into account elective patients; for emergency patients we assume a reservation policy. The flow of elective patients in the Thorax Centre is shown in Fig. 1. Most patients are waiting at home for operation and are admitted to the Medium Care unit (MC) one day before operation. Also patients waiting in another hospital are admitted to the MC unit one day before operation. In case the patient stays before the operation in another department of the University Hospital, the patient is taken directly to one of the operating theatres. Children are admitted to the Children's Department before operation. After the operation the patient stays for some days in an Intensive Care unit (IC) within the Thorax Centre, and after recovery he or she may use the Medium Care unit (MC) for a few days either until return home or return to the referring hospital or department inside the hospital. Children will return to the Children's Department after their IC stay. In the sequel we consider the treatment process only within the boundaries of the Thorax Centre. 
Fig. 1 The flow of elective patients in the Thorax Centre

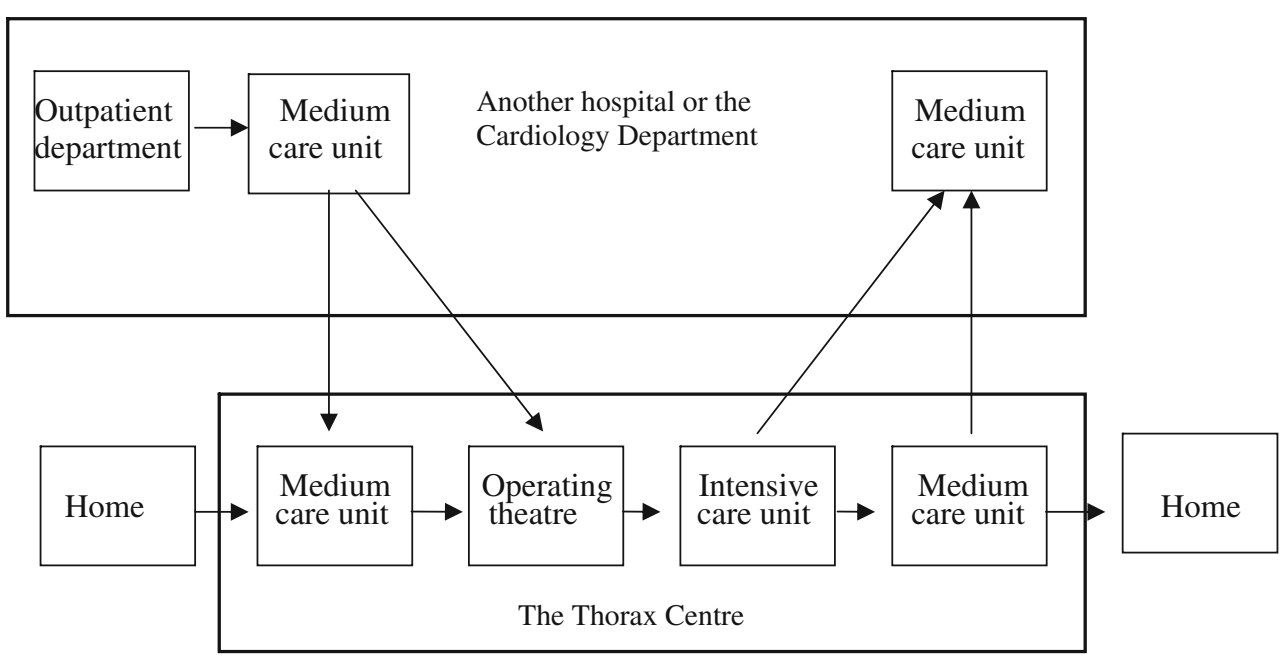

The current planning has a strong focus on the operating theatre capacity. The cardiothoracic surgeon in charge of the planning of operations thought the planning might be improved by taking also into account all other resources involved. The general feeling was the bottleneck resource was the IC, but that this was not reflected in the way surgical schedules were developed. Taking also other resources into account leads to a more efficient use of the available resources and to an increase on the number of patients treated. We concentrate on the following tactical level planning problem: How can the Thorax Centre develop a master surgical schedule, satisfying certain performance criteria?

Due to the pressure on use of beds within the Thorax Centre, the length of stay has shortened compared to the data from 2000 used in the previous project [11]. Patients are discharged at an earlier stage, and complete their remaining time of recovery in the referring hospital or department outside the Thorax Centre. The previous model was based on a deterministic length of stay at IC and MC, representing the average, because more detailed data were not available. The new model is based on a stochastic length of stay at IC and MC and from that we calculate the expected use of resources. We have used recent data over 2006 for developing the new model.

We concentrate in our approach on four resources: OTtime, IC-beds and IC nursing staff, MC-beds. Other resources such as nursing staff for the $\mathrm{MC}$ and specialist capacity are not considered as limitations for the planning and are left out of the modeling approach, in order to focus the model on the most scarce resources.

We will discuss the data used in the model, and illustrate the features of the setting of the Thorax Centre, in terms of patient groups and target volumes, demand requirements, and available resources.

\subsection{Patient groups, volumes and demand requirements}

Table 1 provides information on the patient groups considered, the expected duration of the operation for each group, the average length of stay at the IC (outliers excluded), and the average number of patients per patient group.

The patient groups were distinguished based on the use of OT and IC resources. For children the distinction was between simple and complex. For adults we used a

Table 1 Patient groups, use of OT and IC and 4-week volumes

\begin{tabular}{|c|c|c|c|c|c|}
\hline \multicolumn{2}{|c|}{ Patient group } & \multirow{2}{*}{$\begin{array}{l}\text { Example procedures } \\
\text { Closure ventricular septal defect }\end{array}$} & \multirow{2}{*}{$\frac{\text { Operation duration (h) }}{4}$} & \multirow{2}{*}{$\frac{\text { IC-stay (days) }}{1.1}$} & \multirow{2}{*}{$\frac{\text { \# patients }}{8}$} \\
\hline 1 & Child simple & & & & \\
\hline 2 & Child complex & Arterial switch operation & 8 & 1.1 & 10 \\
\hline 3 & Adult, short OT, short IC & Coronary bypass operation (CABG) & 4 & 1.3 & 75 \\
\hline 4 & Adult, long OT, short IC & Mitral valve plasty & 8 & 1.5 & 14 \\
\hline 5 & Adult, short OT, middle IC & CABG, with expected medium IC stay & 4 & 1.6 & 3 \\
\hline 6 & Adult, long OT, middle IC & Heart transplant & 8 & 4 & 2 \\
\hline 7 & Adult, long OT, long IC & Thoraco-abdominal aneurysm, ELVAD & 8 & 7 & 1 \\
\hline 8 & Adult, very short OT, no IC & Cervical mediastinoscopy & 2 & 0.2 & 8 \\
\hline
\end{tabular}


Table 2 Demand requirements per patient group of OT, IC-stay (real data and rounded to nearest integer), MC-stay (pre-operative days and postoperative days based on real data and rounded) and IC nursing workload per day

\begin{tabular}{|c|c|c|c|c|c|c|c|c|c|c|c|}
\hline \multicolumn{2}{|c|}{ Patient group } & \multirow{2}{*}{$\begin{array}{l}\text { Operation } \\
\text { Duration (h) }\end{array}$} & \multicolumn{2}{|c|}{ IC-stay (days) } & \multicolumn{3}{|c|}{ MC-stay (days) } & \multicolumn{4}{|c|}{ IC nursing (h) per day } \\
\hline & & & Avg & Rounded & Pre-op avg & Post-op avg & Post-op rounded & 1 & 2 & 3 & $>3$ \\
\hline 1 & Child simple & 4 & 1.1 & 1 & 0 & 1.5 & 2 & 12 & 12 & 12 & 12 \\
\hline 2 & Child complex & 8 & 1.1 & 1 & 0 & 1 & 1 & 12 & 12 & 12 & 12 \\
\hline 3 & Adult, short OT, short IC & 4 & 1.3 & 1 & 1 & 5.9 & 6 & 12 & 12 & 12 & 12 \\
\hline 4 & Adult, long OT, short IC & 8 & 1.5 & 2 & 1 & 7.4 & 7 & 12 & 12 & 12 & 12 \\
\hline 5 & Adult, short OT, middle IC & 4 & 1.6 & 2 & 1 & 8.7 & 9 & 12 & 24 & 12 & 12 \\
\hline 6 & Adult, long OT, middle IC & 8 & 4 & 4 & 1 & 15.3 & 15 & 12 & 24 & 12 & 12 \\
\hline 7 & Adult, long OT, long IC & 8 & 7 & 7 & 1 & 9 & 9 & 12 & 24 & 24 & 12 \\
\hline 8 & Adult, very short OT, no IC & 2 & 0.2 & 0 & 1 & 2.3 & 2 & 3 & 3 & 3 & 3 \\
\hline
\end{tabular}

classification of short/long OT use and short/medium/long IC use. By linking the diagnoses of patients to this resource based grouping we were able to define the number of patients that need to be scheduled per planning period of 4 weeks for producing annual volumes of patients per group as agreed upon. The translation from annual numbers to 4week period numbers was done in cooperation with the cardiothoracic surgeon-planner, rounding up to numbers that were seen as a representative 4-weeks caseload.

The operation durations listed in Table 1 are not based on real (detailed) data, but on medical guidelines. This is justified, since the operation durations in our study are only used to determine the number of scheduled operations per day and we are not interested in overrunning of surgery sessions at operational level. For patient group 7 sufficient data on length of stay was missing. Therefore we used instead the length of stay according to the medical guidelines. Table 2 provides information on the demand requirements of each group. Next to the operation duration and the mean length of stay on the IC, the average number of days at the MC, before and after the operation is mentioned, and the estimated number of hours required for IC nursing for the days at the IC.
The last column provides information on the amount of work generated by a patient from a patient group on each day of the stay. The data are based on guidelines used for IC staffing requirements per procedure. We constructed together with the head of the IC a nursing workload profile for the patient group over the days. For procedures with a middle and long IC stay we assume that the nursing workload at the IC is highest at the day of operation (though that might be on average only halve a day) and one or two of the days after. For patient group 7 with missing length of stay data we used again the length of stay according to the medical guidelines. Note that not all children will immediately return to the Children's Department after their IC stay.

\subsection{Length of stay}

In the previous model for the Thorax Centre [11] we used a deterministic length of stay for the IC and the MC, representing the (rounded) average. In the current model we will use a stochastic length of stay for IC and MC, based on empirical data of 2006. The total sample population consists of 593 patients, distributed as 42

Table 3 Length of stay distribution at IC per patient group (based upon sample of 593 patients)

\begin{tabular}{|c|c|c|c|c|c|c|c|c|c|c|c|}
\hline \multirow[t]{2}{*}{ Patient group } & \multicolumn{11}{|c|}{ Probability of length of stay IC (days) } \\
\hline & 0 & 1 & 2 & 3 & 4 & 5 & 6 & 7 & 8 & 9 & 10 \\
\hline 1 Child simple & 0.07 & 0.87 & 0.02 & 0.02 & 0.02 & 0 & 0 & 0 & 0 & 0 & 0 \\
\hline 2 Child complex & 0 & 0.90 & 0.08 & 0.02 & 0 & 0 & 0 & 0 & 0 & 0 & 0 \\
\hline 3 Adult, short OT, short IC & 0.01 & 0.83 & 0.11 & 0.03 & 0.01 & 0.01 & 0 & 0 & 0 & 0 & 0 \\
\hline 4 Adult, long OT, short IC & 0 & 0.83 & 0.10 & 0.04 & 0 & 0.01 & 0.01 & 0 & 0 & 0 & 0.01 \\
\hline 5 Adult, short OT, middle IC & 0 & 0.79 & 0.07 & 0.07 & 0 & 0 & 0 & 0.07 & 0 & 0 & 0 \\
\hline 6 Adult, long OT, middle IC & 0 & 0 & 0.14 & 0.44 & 0.14 & 0.14 & 0 & 0 & 0.14 & 0 & 0 \\
\hline 7 Adult, long OT, long IC & 0 & 0 & 0 & 0 & 0 & 0 & 0 & 1 & 0 & 0 & 0 \\
\hline 8 Adult, very short OT, no IC & 0.79 & 0.21 & 0 & 0 & 0 & 0 & 0 & 0 & 0 & 0 & 0 \\
\hline
\end{tabular}


Table 4 Length of stay distribution at MC per patient group (based upon sample of 593 patients)

\begin{tabular}{lllllllllllll}
\hline & \multicolumn{1}{l}{ Patient group } & \multicolumn{1}{l}{ Probability of length of stay post-operative MC (days) } & & \\
\cline { 2 - 12 } & 0 & 1 & 2 & 3 & 4 & 5 & 6 & 7 & 8 & 9 & 10 & $>10$ \\
\hline 1 Child simple & 0.74 & 0 & 0 & 0 & 0.02 & 0.1 & 0.07 & 0.05 & 0.02 & 0 & 0 & 0 \\
2 Child complex & 0.83 & 0 & 0 & 0 & 0 & 0 & 0.04 & 0.04 & 0.02 & 0.02 & 0 & 0.05 \\
3 Adult, short OT, short IC & 0 & 0.01 & 0.01 & 0.04 & 0.32 & 0.24 & 0.12 & 0.09 & 0.05 & 0.03 & 0.04 & 0.05 \\
4 Adult, long OT, short IC & 0.03 & 0 & 0 & 0.01 & 0.12 & 0.16 & 0.18 & 0.15 & 0.10 & 0.04 & 0.04 & 0.17 \\
5 Adult, short OT, middle IC & 0 & 0 & 0 & 0 & 0.07 & 0.07 & 0.07 & 0.20 & 0 & 0.20 & 0.20 & 0.19 \\
6 Adult, long OT, middle IC & 0 & 0 & 0 & 0 & 0 & 0 & 0 & 0.14 & 0 & 0 & 0.14 & 0.72 \\
7 Adult, long OT, long IC & 0 & 0 & 0 & 0 & 0 & 0 & 0 & 0 & 0 & 0 & 1 & 0 \\
8 Adult, very short OT, no IC & 0.21 & 0.3 & 0.08 & 0.15 & 0.13 & 0.05 & 0 & 0.05 & 0 & 0.03 & 0 & 0 \\
\hline
\end{tabular}

patients of group 1, 53 of group 2, 369 of group 3,68 of group 4, 15 of group 5, 7 of group 6 and 39 of group 8; the available data for each patient in each group consists of the number of days spent at the IC and the number of days spent at the MC. Note that the numbers of sample patients in groups 5 and 6 are limited, possibly yielding inaccurate estimates of the length of stay distributions. However, their share in the total resource requirement is small, and thus we expect these inaccuracies to have little effect on the performance of the operation schedule. To investigate the sensitivity of the operation schedule with respect to the length of stay distributions, we will compare in Section 4.3 the performance of the schedules obtained by using the empirical distributions and by using parameterized distributions fitted to the sample mean and sample standard deviation of the length of stay.

Table 3 provides information on the length of stay distribution at the IC for the patient groups. Table 4 does the same for the MC. The tables clearly illustrate that lengths of stay are far from deterministic.

As the empirical distributions are depending on a rather small sample, we will compare the results with the ones determined by using fitted distributions. This will be described in Section 4.3.

\subsection{Available resources}

Table 5 defines the available capacity for each of the resources per day of the week, and the target utilization level. Defining a level of utilization lower than $100 \%$ allows for dealing with emergencies and fluctuations in number of patients. The data apply to every week in the planning period.

For the Operating Theatres there are four theatres available for $9 \mathrm{~h}$ per day. From the total of $36 \mathrm{~h}$ of capacity available per day $29 \mathrm{~h}$ are aimed to be used by electives, while the rest is reserved for emergencies. On Friday the target utilization is lower. The target utilizations correspond with an average occupancy level by elective patients during the office hours of the Operating Theatres of $80 \%$. The IC unit has ten beds available throughout the working week and four beds during the weekend. The target utilization level for the IC by electives is seven beds throughout the working week and two during the weekend. This corresponds with an average occupancy of the IC of $65 \%$. The MC has 36 beds available every day and the target utilization by electives is 27 beds throughout the whole week. This corresponds with an average occupancy of the MC of $75 \%$. The available IC nursing staff and target

Table 5 Available capacity of resources per day of the week and target utilization levels

\begin{tabular}{|c|c|c|c|c|c|c|c|c|}
\hline \multirow[t]{2}{*}{ Day } & \multicolumn{2}{|l|}{ OT hours } & \multicolumn{2}{|l|}{ IC beds } & \multicolumn{2}{|l|}{ MC beds } & \multicolumn{2}{|c|}{ IC nursing hours } \\
\hline & Capacity & Target & Capacity & Target & Capacity & Target & Capacity & Target \\
\hline Monday & 36 & 29 & 10 & 7 & 36 & 27 & 133 & 91 \\
\hline Tuesday & 36 & 29 & 10 & 7 & 36 & 27 & 133 & 91 \\
\hline Wednesday & 36 & 29 & 10 & 7 & 36 & 27 & 133 & 91 \\
\hline Thursday & 36 & 29 & 10 & 7 & 36 & 27 & 133 & 91 \\
\hline Friday & 36 & 25 & 10 & 7 & 36 & 27 & 133 & 91 \\
\hline Saturday & 0 & 0 & 4 & 2 & 36 & 27 & 52 & 26 \\
\hline Sunday & 0 & 0 & 4 & 2 & 36 & 27 & 52 & 26 \\
\hline
\end{tabular}


Table 6 Expected demand of resources per patient and per 4 weeks, based on real and rounded data

\begin{tabular}{|c|c|c|c|c|c|c|c|c|c|c|c|}
\hline \multirow[t]{3}{*}{ Patient group } & \multirow{3}{*}{$\begin{array}{l}\text { number per } \\
4 \text { weeks }\end{array}$} & \multicolumn{5}{|c|}{ Demand per patient } & \multicolumn{5}{|c|}{ Demand per 4 weeks } \\
\hline & & \multirow[t]{2}{*}{ OT } & \multicolumn{2}{|l|}{ IC } & \multicolumn{2}{|c|}{ Post-op MC } & \multirow[t]{2}{*}{ OT } & \multicolumn{2}{|l|}{ IC } & \multicolumn{2}{|c|}{ MC total } \\
\hline & & & Avg & Rounded & Avg & Rounded & & Avg & Rounded & Avg & Rounded \\
\hline 1 & 8 & 4 & 1.1 & 1 & 1.5 & 2 & 32 & 8.6 & 8 & 12.2 & 16 \\
\hline 2 & 10 & 8 & 1.1 & 1 & 1.7 & 2 & 80 & 11.1 & 10 & 17.0 & 20 \\
\hline 3 & 75 & 4 & 1.3 & 1 & 5.9 & 6 & 300 & 95.1 & 75 & 518.4 & 525 \\
\hline 4 & 14 & 8 & 1.5 & 2 & 7.4 & 7 & 112 & 21.0 & 28 & 117.6 & 112 \\
\hline 5 & 3 & 4 & 1.6 & 2 & 8.7 & 9 & 12 & 4.8 & 6 & 29.0 & 30 \\
\hline 6 & 2 & 8 & 4.0 & 4 & 15.3 & 15 & 16 & 8.0 & 8 & 32.6 & 32 \\
\hline 7 & 1 & 8 & 7.0 & 7 & 9.0 & 9 & 8 & 7.0 & 7 & 10.0 & 10 \\
\hline 8 & 8 & 2 & 0.2 & 0 & 2.3 & 2 & 16 & 1.6 & 0 & 26.4 & 24 \\
\hline Total & 121 & & & & & & 576 & 157.2 & 142 & 763.2 & 769 \\
\hline \multicolumn{7}{|l|}{ Target capacity } & 564 & \multicolumn{2}{|c|}{156} & \multicolumn{2}{|c|}{756} \\
\hline
\end{tabular}

utilization of the IC nursing workload (in number of hours per day) is matched with the number of IC beds. The targets for IC-beds, MC-beds and IC-nursing are defined at a lower level compared to the target for OT hours, to deal with fluctuations in the number of patients.

\subsection{Rough cut capacity check}

We now show what occupancies can be expected when modeling the system, by a simple rough cut capacity check. In Table 6 we provide data on the demand of resources that can be expected with the number of patients scheduled for each planning period of 4 weeks.

Table 6 illustrates that with the current volumes of patients and length of stay, and a reservation of buffer capacity for emergency patients and fluctuations in the load of elective patients, the demand of the resources OT, IC and $\mathrm{MC}$ is in accordance with the target capacity.

The model will help to get a more precise insight into the effect of the reservation of buffer capacity on the perfor-

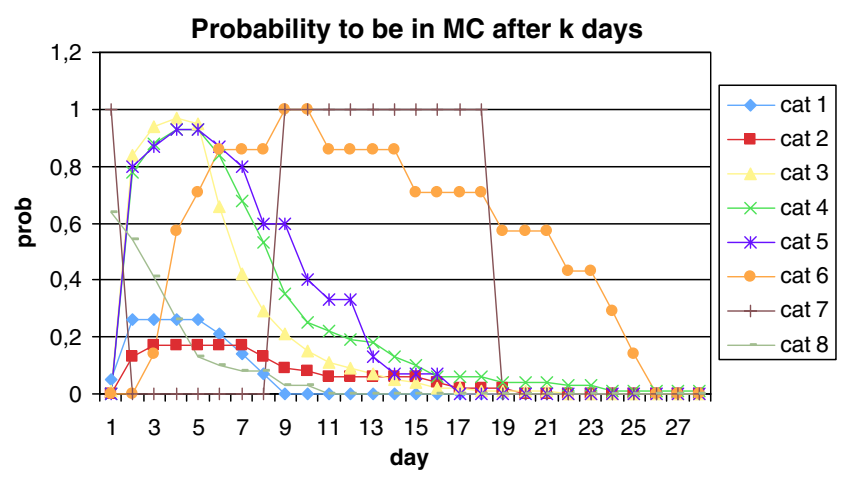

Fig. 2 Probabilities $p_{m c, c, t}$ for the patient groups mance of the system, i.e., for each resource and each day of the planning horizon, the model will predict when the expected utilization will exceed or stay (far) below the specified target.

\subsection{Research question}

One may notice that the length of stay distributions at IC and MC clearly show that there is much variation (see Tables 3, 4 and Fig. 2). In the previous study we only used an estimate for the average length of stay. So the question arises what the effect on the master surgical schedule would be by using the complete distributions of the length of stay instead of only the averages. From these distributions we can then calculate the expected use of each of the resource types. We expect that when we use the complete distribution of the length of stay (referred to as the "stochastic model') our calculations for the expected use of the resources will be much more accurate than when we use only the average length of stay (referred to as the 'deterministic model') and therefore we expect that it allows us to get closer to the target levels.

The central question to be answered by this research is: What is the impact of using stochastic lengths of stay for IC and $\mathrm{MC}$ on generating the cyclic master surgical schedule, which realizes the target utilizations for all resources as close as possible, compared to only using the (rounded) average lengths of stay?

We may limit ourselves to the utilization of resources as we concentrate on the master surgical schedule at tactical level. Therefore we will in this paper not have to deal with waiting times of patients (as the volume of patients used in the master schedule will balance the demand) and with overrun time of surgery schedules (as we concentrate on the master schedule and not on operational schedules). 


\section{Formulation of the optimization problem}

In this section we translate the planning problem into a mathematical problem. Recall that the term 'stochastic model' refers to our length-of-stay distribution only and not to the mathematical problem which is, in fact, deterministic. Let $C$ denote the number of patient categories and $T$ the length of the cyclic operation schedule. On each day of the operation schedule we have to decide on the number and mix of patients to be operated. Hence, the important decision variables are $X_{c, t}$ denoting the number of patients from category $c$ operated on day $t$ of the operation schedule, where $c=1,2, \ldots, C$ and $t=1,2, \ldots, T$. The objective is to determine the variables $X_{c, t}$ satisfying certain constraints and for which the expected utilization of all resources matches the target as close as possible. Below we first formulate the constraints for the variables $X_{c, t}$ and then the objective function.

The total number of patients of group $c$ to be operated in the $T$-days period should be equal to the target patient throughput $T P T_{c}$. Hence,

$$
\sum_{t=1}^{T} X_{c, t}=T P T_{c}, \quad c=1, \ldots, C
$$

To describe the constraints for the utilization of the resources we introduce the parameters $C_{r, t}$ and $U_{r, t}$ indicating the available capacity and target utilization, respectively, of resource $r$ on day $t$, where $r \in R=\{$ ot, ic, $m c, n h\}$. Let the auxiliary variables $U U_{r, t}$ and $O U_{r, t}$ denote the under- and over-utilization (with respect to the target). Then we get for the utilization of operating theatre,

$$
\begin{aligned}
& U_{o t, t}-U U_{o t, t} \leq \sum_{c=1}^{C} O_{c} X_{c, t} \leq U_{o t, t}+O U_{o t, t}, \\
& t=1, \ldots, T
\end{aligned}
$$

where $O_{c}$ denotes the operating theatre time (in hours) required for a category $c$ patient. To formulate the constraints for the expected utilization of the IC unit we introduce the probabilities $p_{i c, c, t}$ denoting the probability that a patient from category $c$ is (still) at the IC unit $t$ days after operation, $t=0,1,2, \ldots$. Then the expected utilization of the IC unit should satisfy

$$
\begin{aligned}
& U_{i c, t}-U U_{i c, t} \leq \sum_{c=1}^{C} \sum_{s=0}^{\infty} p_{i c, c, s} X_{c, t-s} \leq U_{i c, t}+O U_{i c, t} \\
& t=1, \ldots, T
\end{aligned}
$$

In the above constraints we used the convention that the subscript $t-s$ in $X_{c, t-s}$ should be treated modulo $T$ : day 0 is the same as day $T$, day-1 is the same as day $T-1$ and so on. If $n w_{c, t}$ denotes the IC nursing load (in hours) of a category $c$ patient $t$ days after operation, then we get for the expected nursing workload,

$$
\begin{aligned}
& U_{n h, t}-U U_{n h, t} \leq \sum_{c=1}^{C} \sum_{s=0}^{\infty} n w_{c, t} p_{i c, c, s} X_{c, t-s} \leq U_{n h, t}+O U_{n h, t}, \\
& t=1, \ldots, T .
\end{aligned}
$$

Similarly, for the expected utilization of the MC unit we get

$$
\begin{aligned}
& U_{m c, t}-U U_{m c, t} \leq \sum_{c=1}^{C} \sum_{s=1}^{p o_{c}} X_{c, t+s}+\sum_{c=1}^{C} \sum_{s=0}^{\infty} p_{m c, c, s} X_{c, t-s} \\
& \leq U_{m c, t}+O U_{m c, t}, \\
& t=1, \ldots, T,
\end{aligned}
$$

where $p o_{c}$ is the number of pre-operative days at the $\mathrm{MC}$ for category $c$ patients and $p_{m c, c, t}$ is the probability that a patient from category $c$ is at the $\mathrm{MC}$ unit $t$ days after operation, $t=0,1,2, \ldots$ Further, for each of the resources, the available capacity should not be exceeded, so

$U_{r, t}+O U_{r, t} \leq C_{r, t}, \quad r \in R, \quad t=1, \ldots, T$.

In addition to the constraints for the utilization of the resources we have to take into account restrictions valid for specific days of the operation schedule, such as, the number of operations for certain categories of patients is prescribed and fixed (for instance when a cardiothoracic surgeon specialized in children is required which is only available at certain days), or the number of operations for certain combinations of patient categories is limited (for instance to limit combinations of patients which all require maximum IC capacity). The first restriction means that the variables $X_{c, t}$ are prescribed for certain categories $c$ and days $t$. To formulate the second restriction mathematically, let $S$ be a subset of the patient categories and let $B_{t}$ denote the maximum number of patients from the categories $c \in S$ that can be operated on day $t$ of the operation schedule. Then we have to require that

$\sum_{c \in S} X_{c, t} \leq B_{t}, \quad t=1, \ldots, T$

The objective is to minimize the weighted sum of expected under- and over-utilization,

$\sum_{r \in R} w_{r} \sum_{t=1}^{T}\left(U U_{r, t}+O U_{r, t}\right)$

where the weight $w_{r}$ for resource $r$ is defined as

$w_{r}=\frac{a_{r}}{\sum_{t=1}^{T} U_{r, t}}$, 
Table 7 Input parameters and variables

\begin{tabular}{|c|c|c|c|}
\hline $\begin{array}{l}\text { Input } \\
\text { parameters }\end{array}$ & Description & Variables & Description \\
\hline$T$ & Cycle length (days) & $X_{c, t}$ & $\begin{array}{l}\text { Number of category } c \text { patients operated on } \\
\text { day } t\end{array}$ \\
\hline$T P T_{c}$ & Target patient throughput of category $c$ patients & $U U_{r, t}$ & Under-utilization of resource $r$ on day $t$ \\
\hline$C_{r, t}$ & Available capacity of resource $r$ on day $t$ & $O U_{r, t}$ & Over-utilization of resource $r$ on day $t$ \\
\hline$U_{r, t}$ & Target utilization of resource $r$ on day $t$ & & \\
\hline$p_{c}$ & Number of pre-operative days at the MC of category $c$ patient & & \\
\hline$O_{c}$ & Operation duration $(\mathrm{h})$ of category $c$ patient & & \\
\hline$p_{i c}, c, t$ & Probability that $t$ days after operation a category $c$ patient is at the IC & & \\
\hline$p_{m c}, c, t$ & Probability that $t$ days after operation a category $c$ patient is at the MC & & \\
\hline$n w_{c, t}$ & IC nursing workload of category $c$ patient $t$ days after operation & & \\
\hline$w_{r}$ & Relative weight of resource $r$ & & \\
\hline$B_{t}$ & $\begin{array}{l}\text { Maximum number of patients from categories that can be operated on } \\
\text { day } t\end{array}$ & & \\
\hline
\end{tabular}

where $a_{r}$ is some nonnegative number such that the normalized weights sum up to 1 . The weight represents the importance of the resource according to the stake holders.

In Table 7 we summarize the input parameters and variables. The number of integer variables is $C T$ and the number of continuous variables $2 R T$. The number of constraints in the above optimization problem is of order $R T$.

Our planning problem can now be formulated as the following mixed integer linear programming problem:

Minimize $\sum_{r \in R} w_{r} \sum_{t=1}^{T}\left(U U_{r, t}+O U_{r, t}\right)$

Subject to

$$
\begin{aligned}
& \sum_{t=1}^{T} X_{c, t}=T P T_{c}, \quad c=1, \ldots, C \\
& U_{o t, t}-U U_{o t, t} \leq \sum_{c=1}^{C} O_{c} X_{c, t} \leq U_{o t, t}+O U_{o t, t}, \\
& \quad t=1, \ldots, T, \\
& U_{i c, t}-U U_{i c, t} \leq \sum_{c=1}^{C} \sum_{s=0}^{\infty} p_{i c, c, s} X_{c, t-s} \leq U_{i c, t}+O U_{i c, t}, \\
& t=1, \ldots, T, \\
& \quad \leq U_{m c, t}+O U_{m c, t}, \\
& t=1, \ldots, T,
\end{aligned}
$$

$$
\begin{aligned}
& U_{n h, t}-U U_{n h, t} \leq \sum_{c=1}^{C} \sum_{s=0}^{\infty} n w_{c, t} p_{i c, c, s} X_{c, t-s} \leq U_{n h, t}+O U_{n h, t}, \\
& t=1, \ldots, T, \\
& U_{r, t}+O U_{r, t} \leq C_{r, t}, \quad r \in R, \quad t=1, \ldots, T, \\
& \sum_{c \in S} X_{c, t} \leq B_{t}, \quad t=1, \ldots, T, \\
& X_{c, t} \in\{0,1,2, \ldots\}, \quad c=1, \ldots, C, \quad t=1, \ldots, T \\
& \begin{array}{c}
O U_{r, t} \geq 0, \quad O U_{r, t} \geq 0, \quad r \in R, \quad t=1, \ldots, T .
\end{array}
\end{aligned}
$$

Remark 1: Formal description of the probabilities $p_{i c, c, t}$ and $p_{m c}, c, t$

Let the random variables $L_{i c, c}$ and $L_{m c, c}$ denote the length of stay of a patient from category $c$ at the IC and MC unit. Then

$p_{i c, c, t}=P\left(L_{i c, c}>t\right)$

and

$p_{m c, c, t}=P\left(L_{i c, c}+L_{m c, c}>t \mid L_{i c, c} \leq t\right)$.

Hence, $\left\{p_{i c, c, t}, t=0,1,2, \ldots\right\}$ is the complementary distribution of the length of stay at the IC. Clearly, $p_{m c}, c, t$ is 
Table 8 Absolute and relative weights per resource

\begin{tabular}{lcl} 
Resource & Absolute weight $a$ & Relative weight $w$ \\
\hline OT hours & 8 & 0.167 \\
IC beds & 10 & 0.756 \\
MC beds & 3 & 0.047 \\
IC nursing & 5 & 0.029 \\
\hline
\end{tabular}

a conditional probability, requiring the joint probability distribution of the random variables $L_{i c, c}$ and $L_{m c}, c$.

Remark 2: Empirical estimation of $p_{i c, c, t}$ and $p_{m c, c, t}$

The probabilities $p_{i c}, c$, t and $p_{m c}, c, t$ can be directly estimated from data at individual patient level. As mentioned in Section 2.2, the length of stay at IC and $\mathrm{MC}$ is known for each patient. Hence, based on the data, the joint probability distribution of the length of stay at IC and MC can be estimated, and thus also the probabilities $p_{i c, c, t}$ and $p_{m c, c, t}$. In Fig. 2 we show the empirical probabilities $p_{m c, c, t}$ for all patient groups.

Figure 2 shows that the patient groups have distinctive profiles and that there is a high variability between groups in arrival time at the MC.

\section{Implementation and results}

\subsection{Implementation}

The mathematical model is implemented in CPLEX [14]. Solving the deterministic model to optimality did not take much computation time, but the stochastic model could not be solved to optimality. After $24 \mathrm{~h}$ of computation time, there is still a $12 \%$ optimality gap. Still, we think that our solution is quite good, as during the last $9 \mathrm{~h}$ no improvement was found, nor a gap reduction. The model uses as input data the throughput per patient group over the planning period (Table 1), the average operation duration per patient group (Table 1), the length of stay distributions for the IC and MC (Tables 3 and 4 and Fig. 2), and the nursing workload profile (Table 2 combined with Table 3 ). However, to solve the mathematical model, also information is required on the importance of the different resources in the optimization process. Table 8 lists the weights used for reflecting the relative importance of the resources, according to the participants in the project. In other studies, like [13], the weights have been obtained by using a DEA approach, whereas others considered stochastic weight values to represent uncertainty in their value. Our estimations, however, are based on calculations of the additional
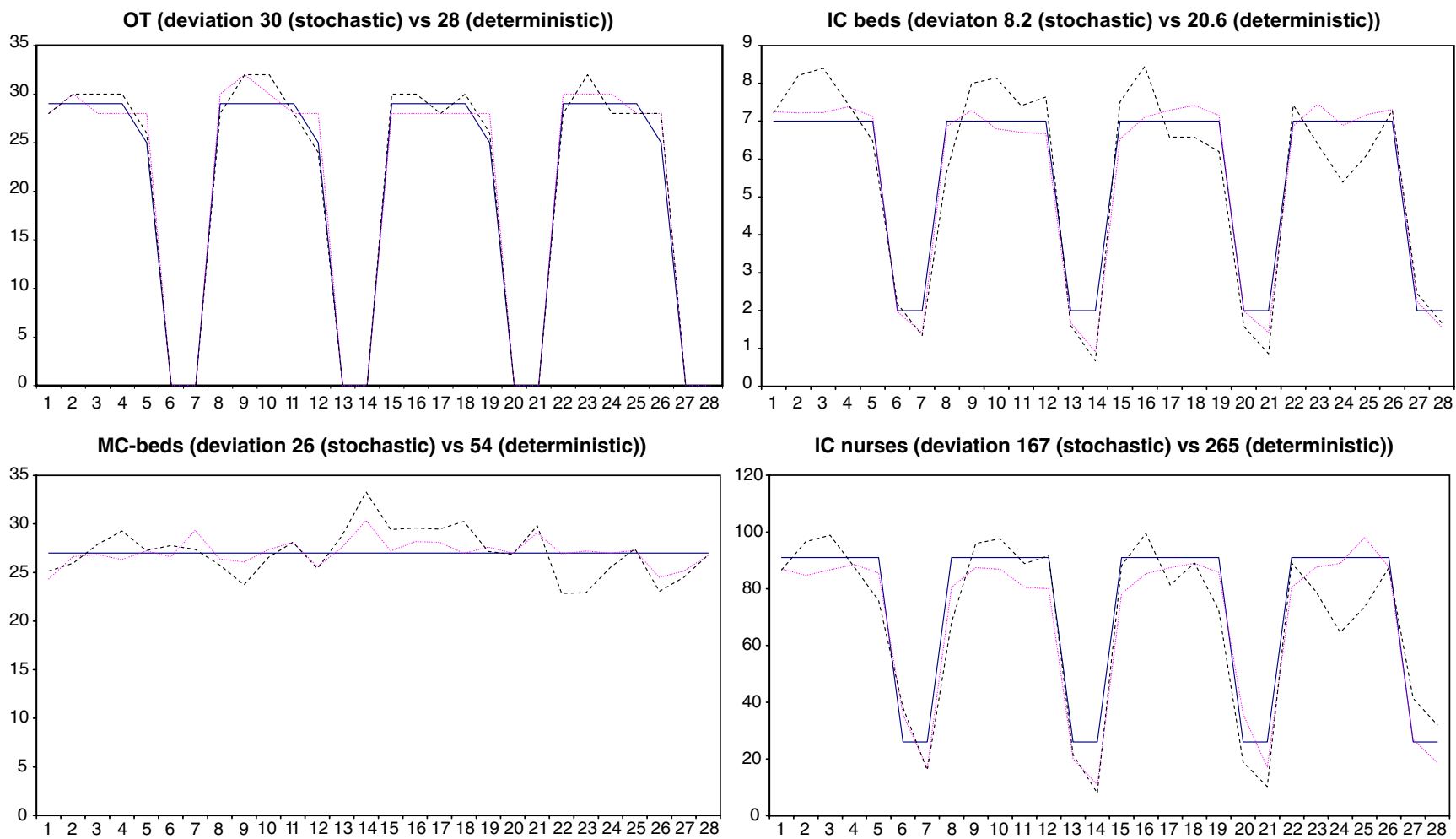

Fig. 3 Comparison of performance with stochastic and deterministic lengths of IC and MC stay (target level in blue, stochastic realization in pink dots, deterministic realization in black dashes) 
Table 9 Difference between realized and target utilizations for the stochastic and the deterministic data model

\begin{tabular}{lcc}
\hline Resource & \multicolumn{2}{c}{ Performance evaluation } \\
\cline { 2 - 3 } & Stochastic & Deterministic \\
\hline OT-hours & 30 & 28 \\
IC-beds & 8.22 & 20.64 \\
MC-beds & 26.38 & 53.66 \\
IC-nursing & 166.56 & 264.66 \\
Overall score & 17.33 & 30.52 \\
\hline
\end{tabular}

costs of an extra unit of resource capacity (including organizational efforts) in the Thorax centre.

As one can see, operating theatre time and IC-bed use are considered very important; IC-nursing and MC-bed use are considered less important. This implies that the model will give higher priority to optimizing the use of IC beds and operating theatres relative to optimizing the use of IC nursing and $\mathrm{MC}$ beds.

\subsection{The impact of stochastic lengths of stay}

Figure 3 illustrates the results obtained by using the deterministic lengths of stay at IC and MC and by using the stochastic lengths of stay. We used as input for the model the data discussed in Section 3. For the deterministic lengths of IC and MC stay we used the rounded data, as shown in Table 2. For the stochastic lengths of IC and MC stay we used the data from Tables 3 and 4. Based upon these data, we determined the best 4-week schedule for the deterministic data and the best 4-week schedule for the stochastic data; so both the deterministic model and the stochastic model were solved to (near) optimality (by application of CPLEX). At first sight, the two schedules look quite similar. However, when we measure the performance of the 4-week cyclic schedules by assuming the stochastic data to be the real ones, we notice quite some differences. The resulting expected

Table 10 Characteristics of the fitted distributions

\begin{tabular}{|c|c|c|c|c|c|c|}
\hline \multirow[t]{2}{*}{ Patient group } & \multicolumn{3}{|l|}{ IC } & \multicolumn{3}{|l|}{$\mathrm{MC}$} \\
\hline & Offset & Mean & Std & Offset & Mean & Stc \\
\hline 1 & 0 & 1.1 & 0.6 & 0 & 1.5 & 2.6 \\
\hline 2 & 1 & 1.1 & 0.4 & 0 & 1.7 & 4 \\
\hline 3 & 1 & 1.3 & 0.9 & 1 & 5.9 & 2.8 \\
\hline 4 & 1 & 1.5 & 1.4 & 0 & 7.4 & 3.8 \\
\hline 5 & 1 & 1.6 & 1.5 & 4 & 8.7 & 2.5 \\
\hline 6 & 2 & 4 & 1.9 & 7 & 15.3 & 5.5 \\
\hline 7 & 4 & 7 & 1.4 & 4 & 10 & 3.4 \\
\hline 8 & 0 & 0.2 & 0.4 & 0 & 2.3 & 2.2 \\
\hline
\end{tabular}

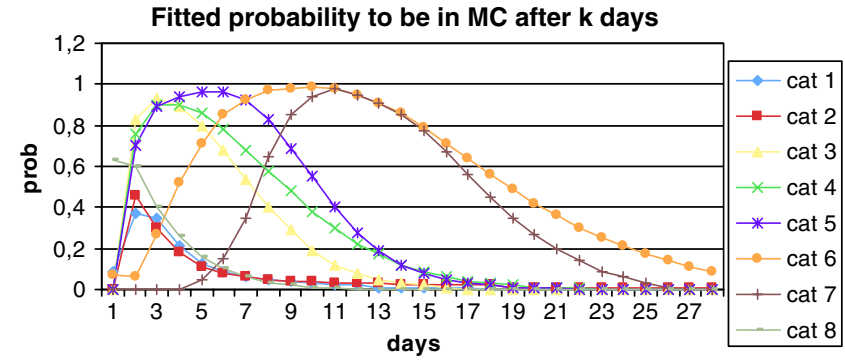

Fig. 4 The fitted probabilities $p_{m c, c, t}$ for the patient groups

utilization levels of resources for both schedules are depicted in Fig. 3. Above each of the graphs we display the total deviation of the expected utilization from its target over the whole planning period for the stochastic versus deterministic model.

The blue line represents the target capacity utilization. The black dashed line refers to the expected utilization level for deterministic lengths of stay and the pink dotted line refers to the expected utilization level for stochastic lengths of stay. We limit ourselves in Fig. 3 to show expected utilization levels, but it is also possible to calculate the corresponding standard deviations. This would illustrate the variability of utilization levels. The results in Fig. 3 demonstrate that the stochastic model performs much better than the deterministic model; for the IC, IC nursing and the $\mathrm{MC}$ the pink dotted lines are more close to the target line than the black dashed ones.

Using the weight function, the score for the combined performance of the resources for the deterministic and the stochastic model are given in Table 9. The total weighted deviation from the target resource levels turns out to be 17.33 when we use stochastic data and 30.52 when we use deterministic data.

Table 9 shows that at the costs of some performance at the OT, big improvements can be obtained for the other resources when we use the stochastic empirical data. This supports the initial feeling of the cardiothoracic surgeon

Table 11 The performance of the fitted and empirical resource use distribution

\begin{tabular}{|c|c|c|c|c|}
\hline \multirow{3}{*}{$\begin{array}{l}\text { Optimization model } \\
\text { Resource }\end{array}$} & \multirow{2}{*}{\multicolumn{2}{|c|}{$\begin{array}{l}\text { Empirical } \\
\text { Performance } \\
\text { evaluation }\end{array}$}} & \multirow{2}{*}{\multicolumn{2}{|c|}{$\begin{array}{l}\text { Fitted } \\
\text { Performance } \\
\text { evaluation }\end{array}$}} \\
\hline & & & & \\
\hline & Empirical & Fitted & Empirical & Fitted \\
\hline OT-hours & 30 & 30 & 30 & 30 \\
\hline IC-beds & 8.22 & 11.21 & 11.92 & 9.69 \\
\hline MC-beds & 26.38 & 34.48 & 39.48 & 32.50 \\
\hline IC-nursing & 166.56 & 182.92 & 139.52 & 125.96 \\
\hline Overall score & 17.33 & 20.44 & 19.95 & 17.54 \\
\hline
\end{tabular}


Table 12 Difference between realized and target utilizations for different OT weights

\begin{tabular}{lccc}
\hline Resource & \multicolumn{2}{l}{ Performance evaluation } \\
\cline { 2 - 4 } & $\begin{array}{l}\text { OT weight } \\
\text { (base case) }\end{array}$ & OT weight 0 & $\begin{array}{l}\text { Only OT } \\
\text { weight }\end{array}$ \\
\hline OT-hours & 30 & 100 & 28 \\
IC-beds & 8.22 & 8.42 & 36.26 \\
MC-beds & 26.38 & 22.22 & 95.02 \\
IC-nursing & 166.56 & 157.28 & 470.36 \\
\hline
\end{tabular}

planner, and is also in line with other studies reported by McManus et al. [7] and Wachtel and Dexter [9].

4.3 Sensitivity analysis for length of stay distribution and weights

As the empirical distributions are depending on a rather small sample, we will compare the results obtained by the empirical distributions to the results obtained by discrete distributions fitted to the sample mean and sample standard deviation of the length of stay at IC and MC, according to the recipe provided in [12], and requiring that the fitted distribution has the same offset as the empirical distribution. The mean, standard deviation and offset of the fitted distributions are displayed in Table 10. Note that, after consulting the surgeons, we artificially introduced a standard deviation for the length of stay of patients in group 7, for which data was missing. Figure 4 shows the resulting probabilities $p_{m c, c, t}$ that have been obtained by combining the fitted IC distribution and the fitted MC distribution, and assuming that the lengths of stay at IC and $\mathrm{MC}$ are independent (thus ignoring possible correlation). Comparison of Figs. 2 and 4 shows that the fitted distributions resemble the empirical ones, but obviously, they are much smoother.

The total performance stays almost the same when we replace the empirical distribution by a fitted one with the same mean and standard deviation for the various resource uses. In Table 11 we show the performance, based on both the fitted and empirical resource use distributions. We consider the distributions both for the decision making (optimization model) as well as for the determination of the performance (performance evaluation). Logically, the best performance is obtained when the decision making is done with the same distribution as the performance measuring, but using a different distribution still gives a reasonably good performance, compared to the poor results for the deterministic distribution in Table 9.

In order to obtain insight into the best possible performance of IC and MC we also considered a scenario with the stochastic data model where the OT weight has been put to 0 and another scenario with the stochastic data model where only the OT has a non-zero weight, a situation that can be compared to the current hospital practice with a strong focus on only OT use optimization (Table 12).

From this table we learn that some improvement is still possible for MC-beds and IC-nurses, but that the improvement is not significant. It also turns out that the possible reduction for OT-hours is very limited. It illustrates once more that the current practice with a strong focus on OT use has a very bad performance impact on the other resources.

\section{Conclusions and recommendations}

In many organizations, the capacity planning is based upon standard durations for the different process phases. Vissers et al. [11] have considered such an approach for determining the optimal patient mix for a cardiothoracic surgery department. In this paper we have extended their model, by considering stochastic durations for the stay in the IC unit and in the MC unit. Based upon a large sample of patients of a Dutch cardiothoracic surgery department, we created an empirical distribution for the durations of the IC phase and the $\mathrm{MC}$ phase and used this in our mixed integer linear programming model, trying to determine a cyclic master operation schedule minimizing weighted deviations between realized and targeted resource use. Introducing the stochastic durations turned out to decrease the deviations by more than $40 \%$. Therefore we believe that it is very important to consider these stochastic durations in generating an optimal operation schedule. The current approach also seems to be robust to small changes in the stochastic durations; the results based on smooth distributions fitted to sample characteristics appeared close to the ones produced by the empirical distributions.

We also looked at the maximum performance for IC and MC units, when the OT would have a very large capacity, and found that only limited further improvement is still possible in these units. When we only focus on the OT-use, the OT performance can be slightly improved, but with disastrous effects for the other resources. Our study demonstrates that the current practice of scheduling surgeries with methods based principally on utilization of operating theatres may not result in an overall good performance of the system. Using a broader scope including perspectives of operating theatres as well as intensive care units and wards results in a better overall performance. This supports findings from previous studies by McManus et al. [7], Wachtel and Dexter [9] and Beliën and Demeulemeester [5] who pointed at the artificial variation in the utilization of resources introduced by 
surgery schedules used in operating theatres that can be avoided by taking into account the dependencies between these resources when developing master schedules.

Future work will be done on the use of these tactical planning results in an operational planning environment and in testing how the operational planning can come close to the tactical planning schedule. A further recommendation is to look at the reservations policies for emergency admissions.

Acknowledgement The authors would like to thank Jully Jeunet for additional CPLEX programming.

Open Access This article is distributed under the terms of the Creative Commons Attribution Noncommercial License which permits any noncommercial use, distribution, and reproduction in any medium, provided the original author(s) and source are credited.

\section{References}

1. Kusters RJ, Groot PMA (1996) Modelling resource availability in general hospitals. Design and implementation of a decision support model. Eur J Oper Res 88:428-445, doi:10.1016/03772217(95)00201-4

2. Vissers JMH, Adan IJBF, Dellaert NP (2007) Developing a platform for comparison of hospital admission systems: an illustration. Eur J Oper Res 180:1290-1301, doi:10.1016/j. ejor.2006.04.034

3. Gemmel P, Van Dierdonck R (1999) Admission scheduling in acute care hospitals: does the practice fit with the theory. Int J Oper Prod Manage 19:863-878, doi:10.1108/01443579910 280188
4. Smith-Daniels VL, Schweikhart SB, Smith-Daniels DE (1988) Capacity management in health care services. Decision Sciences 19:898-919, doi:10.1111/j.1540-5915.1988.tb00310.x

5. Beliën J, Demeulemeester E (2007) Building cyclic master surgery schedules with levelled resulting bed occupancy. Eur J Oper Res 176:1185-1204

6. van Oostrum JM, van Houdenhoven M, Hurink JL, Hans EW, Wullink G, Kazemier G (2008) A master surgical scheduling approach for cyclic scheduling in operating room departments. OR Spectrum 30:355-374, doi:10.1007/s00291-006-0068-x

7. McManus ML, Long MC, Cooper A, Mandell J, Berwick DM, Pagano M et al (2003) Variability in surgical caseload and access to intensive care services. Anesthesiology 98:1491-1496, doi:10.1097/00000542-200306000-00029

8. McManus ML, Long MC, Cooper A, Litvak E (2004) Queuing theory accurately models the need for critical care resources. Anesthesiology 100:1271-1276, doi:10.1097/00000542-20040 5000-00032

9. Wachtel RE, Dexter F (2008) Tactical increases in operating room block time for capacity planning should not be based on utilization. Anesth Analg 106:215-226

10. Adan IJBF, Vissers JMH (2002) Patient mix optimisation in hospital admission planning: a case study. Special issue on 'operations management in health care' of the International Journal of Operations and Production Management 22:445-461.

11. Vissers JMH, Adan IJBF, Bekkers JA (2005) Patient mix optimization in cardiothoracic surgery planning: a case study. IMA J Manage Math 16:281-304, doi:10.1093/imaman/dpi023

12. Adan IJBF, van Eenige MJA, Resing JAC (1995) Fitting discrete distributions on the first two moments. Probab Engrg Inform Sci 9:623-632

13. O'Neill L, Dexter F (2007) Tactical increases in operating room block time based on financial data and market growth estimates from data envelopment analysis. Anesth Analg 104:355-368, doi:10.1213/01.ane.0000253092.04322.23

14. http://www.ilog.com/products/cplex/ 